Modeling of in situ Biorestoration of Organic Compounds in Groundwater

Chen Y. Chiang Clint N. Dawson

Mary F. Wheeler

October, 1990

TR90-31 



\title{
MODELING OF IN-SITU BIORESTORATION OF ORGANIC COMPOUNDS IN GROUNDWATER
}

\author{
Chen Y. Chiang \\ Shell Development Company, Houston, Texas U.S.A.
}

Clint N. Dawson

Department of Mathematics, University of Chicago, Illinois U.S.A.

Mary F. Wheeler

Department of Mathematics, University of Houston, Texas U.S.A.

\begin{abstract}
A convergent numerical method for modeling in situ biorestoration of contaminated groundwater is outlined. This method treats systems of transport-biodegradation equations by operator splitting in time. Transport is approximated by a finite element modified method of characteristics. The biodegradation terms are split from the transport terms and treated as a system of ordinary differential equations. Numerical results for vertical cross-sectional flow are presented. The effects of variable hydraulic conductivity and variable linear adsorption are studied.
\end{abstract}

Keywords: Modified method of characteristics, time-splitting, in situ biorestoration, adsorption, contaminant transport.

\section{Introduction}

In recent years, in situ biorestoration has emerged as the most promising technology for the treatment of contaminated groundwater. Numerous field and laboratory studies have 
indicated that indigenous microorganisms degrade harmful compounds in both aerobic and anaerobic aquifers, and control contaminant movement; see $[14,23,27,28]$ and the references therein. In situ biorestoration involves the enhancement of these natural processes by introducing dissolved oxygen and/or other nutrients into the system. As discussed in a recent Environmental Protection Agency (EPA) report [27], in situ biorestoration is effective for several reasons:

1) it is environmentally sound, generally producing no toxic byproducts,

2) it typically results in complete degradation of contaminants,

3) it utilizes indigenous microflora and does not introduce potentially harmful organisms,

4) it is fast, safe, and generally economical,

and

5) the treatment moves with the groundwater.

An example of the advantage of this process over standard technology is the Ambler, Pennsylvania field site [22]. Here, a gasoline spill was remediated in 10 months by biorestoration, whereas standard "pump and treat" technology was estimated to take 100 years.

The biorestoration process is physically and chemically complex, involving transport and interaction of substrate, dissolved oxygen, and microorganisms. Many factors must be considered, such as the characteristics of the organisms, the primary substrate and nutrients, cometabolic substrates, contaminant concentration and location, available electron acceptors, and environmental conditions, including sorption, ion exchange, the effect of colloidal particles, soil $\mathrm{pH}$ and mineral composition, temperature, rock heterogeneity, and fractures. 
Much of the field and laboratory data used to estimate these effects is incomplete and/or inexact, and is scale-dependent.

Presently, a major research emphasis is in laboratory column and batch reactor experiments and intermediate scale laboratory studies [28] to understand and quantify the sorption and biodegradation kinetics. Because in-depth studies on a field scale are expensive, mathematical modeling serves as a valuable link between the experimental studies and scientific theory. It is especially useful in 1) understanding the mechanisms of interactive transport, sorption, and biodegradation, 2) analyzing the sensitivity and significance of various parameters in a model and providing insight into field data collection activities, and 3) efficiently investigating various strategies for remediation and mitigation.

The governing equations describing these processes are coupled nonlinear partial differential equations involving transport, biodegradation, and sorption of substrates, transport and utilization of dissolved oxygen and other nutrients, and the metabolic dynamics and possible transport of microorganisms. In the next section, we discuss some of the specific mathematical models which have been proposed by Molz, Widdowson, and Benefield [20], Borden and Bedient [1], and Kindred and Celia [14]. In Section 3, we describe a numerical method for approximately solving the governing equations arising from the latter two formulations: In our algorithm, an accurate mixed finite element method is used to calculate the flow field. A time-splitting procedure is then employed to solve the transportbiodegradation equations. Transport is approximated by a modified method of characteristics, and the reactions are treated as a system of ordinary differential equations, which are solved by integration in time. Finally, in Section 4, we present numerical results for vertical cross-sectional flow. In these studies, we are particularly interested in the effects 
of spatially varying hydraulic conductivity, and spatially varying organic carbon content, which influences adsorption.

\section{Mathematical models}

Various mathematical models have been proposed for describing the biodegradation process. In this section we briefly discuss those proposed by Borden and Bedient, Molz, et al, and Kindred and Celia. The latter two models generalize the first; each model includes the biodegradation terms as sources and sinks in the transport equations.

Borden and Bedient, and Molz, et al, have formulated equations for aerobic systems with two nutrients (substrate and dissolved oxygen). Borden and Bedient based their model on the Monod kinetics. This formulation describes degradation of a single organic compound and dissolved oxygen utilization by microbes. Molz, et al, incorporated a biofilm model of Rittman and McCarty [24] which describes the growth, uptake, and degradation of contaminants within a consortium of microbes which are attached to the soil particles. This approach consists of five coupled equations describing substrate transport, dissolved oxygen transport, utilization of substrate and dissolved oxygen by microcolonies, and microcolony evolution, and requires the knowledge of nineteen input parameters. By assuming that substrate and dissolved oxygen utilization are constant multiples of substrate and dissolved oxygen in solution, this model reduces essentially to that of Borden and Bedient.

An extension of these models to include anaerobic biodegradation has been developed by Kindred and Celia [14]. They also generalize the aerobic metabolism with uptake to multiple organic energy substrates, and include cometabolism of nongrowth substrates by 
aerobic bacteria. The Michaelis-Menten model of uptake kinetics is assumed, and uptake inbibition is included.

All of these models treat sorption as a linear equilibrium isotherm and assume no interaction between sorption and biodegradation.

Numerical simulations using these models have been implemented by the above mentioned researchers. Borden and Bedient [1] and Rifai [23] have modified the KonikowBredeheoft [15] code to simulate aerobic biodegradation by assuming an instantaneous stoichiometric biochemical oxidation of the soluble organics. Widdowson, et al [32], employ a first-order, finite difference Eulerian-Lagrangian scheme for transport, and use a NewtonRaphson method to treat the nonlinear reaction terms. Their transport scheme is equivalent to a finite difference modified method of characteristics, as analyzed by Douglas and Russell $[10]$

The one space dimensional numerical experiments by Celia, et al [2], utilize an “optimal test function" approach which is closely related to the $\mathcal{H}^{-1}$ Galerkin method [11]. Biodegradation is treated by quadratic extrapolation in time.

Of the above, Borden and Bedient, Rifai, and Widdowson, et al, have compared their numerical results with field data. In all of the above mentioned simulations, a constant flow field was assumed.

We would also like to mention more recent numerical work in this area by MacQuarrie, et al [16], and MacQuarrie and Sudicky [17].

Another numerical method for approximating the Borden and Bedient equations has been formulated by Wheeler and Dawson [30]. This work represents extensions of earlier work of Douglas and Russell [10], Russell [25], Russell, et al [26], and Chiang, et al [4] to 
transport-biodegradation systems. Numerical experiments utilizing this method have been reported in $[6,7,8,30,31]$. In the next section, we discuss this method in the context of more general biodegradation models.

\section{$3 \quad$ Numerical Method}

In this section, we describe a numerical procedure for solving a general transport-biodegradation model, assuming linear instantaneous adsorption of substrate. This procedure is applicable to the Borden and Bedient model and the various models proposed by Kindred and Celia. We do not discuss application to the formulation of Molz, et al, however, time-splitting may be employed in this context as well.

We denote by $S_{i}, i=1, \ldots, m_{s}$, the concentrations of various substrates in solution; $O$ represents the concentration of dissolved oxygen; $N_{i}, i=1, \ldots, m_{n}$, denotes the concentrations of various other nutrients and electron acceptors (such as nitrate and assimilatory nitrogen), and $B_{i}, i=1, \ldots, m_{b}$, the concentrations of various bacterial species.

Let $\mathcal{S}=\left(S_{1}, \ldots, S_{m_{\mathrm{b}}}\right), \mathcal{N}=\left(N_{1}, \ldots, N_{m_{n}}\right)$, and $\mathcal{B}=\left(B_{1}, \ldots, B_{m_{b}}\right)$. The equations describing biodegradation of substrates, utilization of dissolved oxygen and nutrients, and microbial metabolism can be written in general form as

$$
\begin{aligned}
R_{\mathcal{S}_{i}} \frac{d S_{i}}{d t}=R S_{i}(\mathcal{S}, O, \mathcal{N}, \mathcal{B}), \quad i=1, \ldots, m_{s} \\
\frac{d O}{d t}=R O(\mathcal{S}, O, \mathcal{N}, \mathcal{B}) \\
\frac{d N_{i}}{d t}=R N_{i}(\mathcal{S}, O, \mathcal{N}, \mathcal{B}), \quad i=1, \ldots, m_{n}
\end{aligned}
$$


and

$$
\frac{d B_{i}}{d t}=R B_{i}(\mathcal{S}, O, \mathcal{N}, \mathcal{B}), \quad i=1, \ldots, m_{b}
$$

where $R_{S_{i}}$ is the retardation factor for substrate $i$ due to adsorption. We note that, in certain cases, the right hand side terms may not explicitly depend on all of the variables.

Combining (1)-(3) with linear transport and instantaneous adsorption we obtain the following system of equations,

$$
\begin{gathered}
\phi R_{S_{i}} \frac{\partial S_{i}}{\partial t}-\nabla \cdot\left(D \nabla S_{i}-\mathbf{u} S_{i}\right)=\phi R S_{i}, \quad i=1, \ldots, m_{s}, \\
\phi \frac{\partial O}{\partial t}-\nabla \cdot(D \nabla O-\mathbf{u} O)=\phi R O, \\
\phi \frac{\partial N_{i}}{\partial t}-\nabla \cdot\left(D \nabla N_{i}-\mathbf{u} N_{i}\right)=\phi R N_{i}, \quad i=1, \ldots, m_{n}, \\
\mathbf{u}=-K \nabla h,
\end{gathered}
$$

and

$$
\nabla \cdot \mathbf{u}=0
$$

where $D=D(\mathbf{u})$ is the diffusion/dispersion tensor, which includes the effects of molecular diffusion and longitudinal and transverse dispersion, $u=\left(u_{1}, u_{2}\right)^{T}=$ Darcy velocity, $h=$ hydraulic head, $K=$ hydraulic conductivity, and $\phi=$ porosity. Here we have neglected hydraulic source terms (wells) which may also be included in the system of equations. Combining (5)-(9) and (4) with appropriate initial and boundary conditions completes the mathematical description of the problem. Note that we have assumed microbe transport 
is negligible. If this is not the case, we obtain equations of the form (5) for those species which are transported.

Solving these equations numerically involves the treatment of sharp fronts, heterogeneities, physical dispersion, and time scale effects. In particular, biodegradation generally needs to be approximated on a much smaller time scale than advection and dispersion. All of these difficulties must be handled in a robust and computationally efficient fashion. We feel that the approach described below satisfies these requirements. The algorithm we have implemented is as follows:

(1) Accurate Darcy velocities and head are calculated using a higher-order mixed finite element procedure.

Steps (2) and (3) are repeated $N$ times where $N \Delta t=$ final time:

(2) A finite element modified method of characteristics is used to treat each of the transport equations, i.e., (5)-(7) with right hand sides set to zero.

(3) The biodegradation equations (1)-(4) are treated as a system of ordinary differential equations, where a second-order, explicit Runge-Kutta method is employed, using many small time steps.

In this paper, we consider only two space dimensions; however, the methodology is easily extendable to three space dimensions.

The approximating spaces we use in step 1 are the Raviart-Thomas spaces with $r=1$. That is, the hydraulic head approximation is a discontinuous, piecewise bilinear function on a tensor product, rectangular grid. The $x$-component of velocity ( $y$-component) is approximated by a continuous, piecewise quadratic function in $x(y)$ and a discontinuous, piecewise 
linear function in $y(x)$. The dimension of these spaces is approximately $12 N_{x} N_{y}$ where $N_{x}$ and $N_{y}$ are the number of intervals in the $x$ and $y$ directions respectively; for more details, see [9]. These approximating spaces for hydraulic head and velocity yield higher order convergence rates than the standard cell-centered finite differences (i.e., Raviart-Thomas approximating spaces with $r=0$ ), and do not introduce any grid orientation effects. Moreover, in the mixed finite element method, hydraulic conductivity is treated in an integral formulation, which allows for more accurate modeling of highly varying conductivities. A complete analysis of the mixed formulation and superconvergence results can be found in [21].

The time-splitting algorithm outlined in steps 2 and 3 for solving the transport-biodegradation equations (5)-(7) is most conveniently described by considering a single component, which we denote as $C$. Let $R_{C}$ be the retardation factor for component $C$, with the convention that for non-substrates, $R_{C}=1$, and let $\psi=\phi R_{C}$. Let $\Delta t>0$, and $t^{n}=n \Delta t$, for some integer $n \geq 0$. Assume $C^{n} \equiv C\left(t^{n}\right)$ is known, and $C^{n+1} \equiv C\left(t^{n}+\Delta t\right)$ is desired. Thus, we want to solve

$$
\psi \frac{\partial C}{\partial t}-\nabla \cdot D \nabla C+\mathbf{u} \cdot \nabla C=\phi R C
$$

for $t^{n}<t \leq t^{n+1}$ with initial condition $C^{n}$. We time-split (10), approximating biodegradation and transport sequentially; denote the biodegradation solution by $C^{*}$ and the transport solution by $\bar{C}$. First, we solve the system of ordinary differential equations for biodegradation given by (1)-(4), which has components of the form

$$
R_{C} \frac{d C^{*}}{d t}=R C(\mathcal{S}, O, \mathcal{N}, \mathcal{B})
$$

This system is solved for $t^{n}<t \leq t^{n+1}$ with component initial conditions $C^{*}\left(t^{n}\right)=C^{n}$. Note 
that since microorganisms are not transported, this completes the step for these components. Next, transport of substrate, dissolved oxygen, and other nutrients is modeled by solving equations of the form

$$
\begin{aligned}
\psi \frac{\partial \bar{C}}{\partial t}-\nabla \cdot(D \nabla \bar{C}-\mathbf{u} \bar{C}) & =0, \quad t^{n}<t \leq t^{n+1}, \\
\bar{C}\left(t^{n}\right) & =C^{*}\left(t^{n+1}\right) .
\end{aligned}
$$

This two step procedure gives $\bar{C}\left(t^{n+1}\right)$ which is an approximation to $C^{n+1}$.

We approximate $C^{n}$ for each $n$ in the finite element space of continuous, piecewise bilinears on a rectangular mesh, which we denote as $\mathcal{M}_{h}$. The system of ordinary differential equations corresponding to (11) is solved at each node in the finite element mesh, using an explicit, second-order Runge-Kutta method with time step $\Delta t_{s}$, which is generally much smaller than the transport time step, depending on the stiffness of the system (11). These solutions are interpolated to obtain an approximation to $C^{*}\left(t^{n+1}\right)$ in $\mathcal{M}_{h}$, denoted by $C_{h}^{*}\left(t^{n+1}\right)$

The transport solutions are approximated using a finite element modified method of characteristics (MMOC). Let $(\cdot, \cdot)$ denote the $L^{2}$ inner product on $\Omega$. Applying (9), we rewrite (12) as

$$
\psi \frac{\partial \bar{C}}{\partial t}+\mathbf{u} \cdot \nabla \bar{C} \equiv \sqrt{\psi^{2}+|\mathbf{u}|^{2}} \frac{\partial \bar{C}}{\partial \tau}=\nabla \cdot D \nabla \bar{C}
$$

where $\frac{\partial C}{\partial \tau}$ is a directional derivative. Denote by $\bar{C}_{h}^{n+1} \in \mathcal{M}_{h}$ the approximation to $\bar{C}\left(t^{n+1}\right)$. We determine $\bar{C}_{h}^{n+1}$ by

$$
\left(\psi \frac{\bar{C}_{h}^{n+1}-\overline{\bar{C}}_{h}^{n}}{\Delta t}, v\right)+\left(D \nabla \bar{C}_{h}^{n+1}, \nabla v\right)=0, \quad v \in \mathcal{M}_{h},
$$

where

$$
\overline{\bar{C}}_{h}^{n}(\mathbf{x})=C_{h}^{*}\left(\mathbf{x}-\frac{\mathbf{u}(\mathbf{x})}{\psi(\mathbf{x})} \Delta t, t^{n+1}\right)
$$


Note that (14) is obtained from (13) by integrating by parts and approximating the $\tau$ derivative using backward differencing in time.

For more details on this time-splitting procedure, and for theoretical error estimates, see [30]. The MMOC has been analyzed extensively for transport problems, see [5] and the references therein, and comparisons with analytical solutions can be found in [4].

One major advantage of the MMOC over other transport procedures is that large time steps can be taken, without loss of accuracy or stability, because the time truncation error is $\mathcal{O}\left(\Delta t\left\|c_{\tau \tau}\right\|\right)$, where $\left\|c_{\tau \tau}\right\|$ is the $L^{2}$ norm of the second derivative of the solution in the $\tau$-direction [10]. This term is generally much smaller than the time truncation error for standard second order in time procedures. By using time-splitting, we are able to approximate each piece of the calculation, namely, transport and biodegradation, with an appropriate numerical method, and an appropriate time step. The error analysis given in [30] for the time-splitting procedure discussed here shows that, for sufficiently smooth $C$, the $L^{2}$ norm of the error at any time level is $\mathcal{O}\left(h^{2}+\Delta t+\Delta t_{s}^{2}\right)$.

\section{Numerical results}

The numerical method described above has been applied to the simulation of vertical crosssectional flow. In these studies, we have concentrated on two aspects of the problem, namely, the effects of variable hydraulic conductivity, and the effects of spatially varying retardation. For simplicity, we consider the mathematical model of Borden and Bedient. Thus, we simulate the flow of dissolved oxygen and a single substrate, and the interaction of these components with a single microbial population. 
Biodegradation in this model is assumed to satisfy the Monod kinetics, as given in the following system of ordinary differential equations:

$$
\begin{aligned}
& R_{S} \frac{d S}{d t}=-B \cdot \bar{k} \cdot\left(\frac{S}{K_{S}+S}\right)\left(\frac{O}{K_{O}+O}\right) \equiv R S(O, S, B), \\
& \frac{d O}{d t}=-B \cdot \bar{k} \cdot F \cdot\left(\frac{S}{K_{S}+S}\right)\left(\frac{O}{K_{O}+O}\right) \equiv R O(O, S, B),
\end{aligned}
$$

and

$$
\begin{aligned}
\frac{d B}{d t} & =B \cdot \bar{k} \cdot Y \cdot\left(\frac{S}{K_{S}+S}\right)\left(\frac{O}{K_{O}+O}\right)-b \cdot B+B_{g} \\
& \equiv R B(O, S, B)
\end{aligned}
$$

where $\bar{k}=$ maximum hydrocarbon utilization rate per unit mass microorganisms, $Y=$ microbial yield coefficient, $K_{S}=$ hydrocarbon half saturation constant, $K_{O}=$ dissolved oxygen half saturation constant, $b=$ microbial decay rate, and $F=$ ratio of dissolved oxygen to hydrocarbon consumed. The last term on the right side of (18), $B_{g}$, is a small growth term which assures that microbes do not become extinct. Combining (16)-(18) with $(5),(6),(8)$, and (9) completes the definition of the mathematical model.

As seen in (5), the assumption of linear, instantaneous adsorption gives rise to a retardation factor for substrate, which is given by

$$
R_{S}=1+\rho_{b} K_{d} / \phi
$$

where $\rho_{b}$ is the bulk mass density $\left(g / \mathrm{cm}^{3}\right)$, and $K_{d}$ is the distribution coefficient $\left(\mathrm{cm}^{3} / \mathrm{g}\right)$, see [12]. The latter term can be expressed as

$$
K_{d}=K_{o c} f_{o c}
$$

where $K_{o c}$ is the soil partition coefficient normalized to the organic carbon content of the soil, and $f_{o c}$ is the fraction of organic carbon in the soil. 
Field studies indicate that $f_{o c}$ may vary between $.01 \%$ and $1 \%$ within a field site [3], thus $f_{\text {oc }}$ varies spatially, sometimes by orders of magnitude. Since no precise spatial representation for $f_{o c}$ is known, we will make three different assumptions on the spatial distribution of organic carbon:

1) $f_{o c}$ is uniform.

2) The values of $f_{o c}$ satisfy certain statistical properties; $f_{o c}$ values at various spatial locations were generated stochastically using a code developed by D. Moissis. In this code, the user specifies correlation length, mean, and standard deviation. A spatially correlated, log-normal distribution of values is then generated. Finally, a small amount of random noise is added. For more details on this algorithm, see [19].

3) Values of $f_{o c}$ decrease with depth. This assumption is based on the fact that organic carbon content is proportional to the amount of humic substance in the soil, which is more prevalent near the surface.

The expected effect of varying $f_{\text {oc }}$ spatially is to induce another level of variation into the effective velocity for the substrate.

Karickhoff, et al [13], showed that reasonable partition coefficients $\left(K_{o c}\right)$ could be deduced from the octanol/water partition of the chemical, $K_{\text {ow }}$. They presented the following relationship between $K_{o c}$ and $K_{o w}$,

$$
\log K_{o c}=\log K_{o w}-0.21
$$

Values for $K_{\text {ow }}$ can vary widely, depending on the substrate. For benzene, toluene, and xylene, $K_{\text {ow }}$ varies from $135-1500 \mathrm{~cm}^{3} / \mathrm{gm}[18]$. Moreover, for porosities in the $.2-.4$ 
range, $\rho_{b}$ varies between $1.6-2.1 \mathrm{~g} / \mathrm{cm}^{3}$. Given these ranges of values for $K_{o w}, \rho_{b}$, and $f_{o c}$, the retardation factors for different compounds may vary between 1 and 129 .

In the numerical simulations described below, the physical domain $\Omega$ is a rectangular cross-section of an aquifer, 260 feet long and 6 feet deep. Hydraulic head is specified on the left and right edges of $\Omega$, and no-flow boundary conditions are assumed at the top and bottom; the head drop is 7.5 feet from left to right. Gravitational effects are not included, since density differences are negligible. In the transport equations, concentrations of dissolved oxygen and substrate are specified on the inflow (left) boundary, and at the right boundary we assume

$$
\phi R_{C} C_{t}+\mathbf{u} \cdot \nabla C=0, \quad C=O, S
$$

thus, substrate and dissolved oxygen are transported out of the domain through the right boundary. Substrate concentration is assumed to be zero at the inflow boundary, and dissolved oxygen concentration is given by

$$
O(x=0, t)= \begin{cases}t / 20(\text { days }) * 40 \mathrm{mg} / 1, & 0 \leq t \leq 20 \\ 40 \mathrm{mg} / \mathrm{l}, & \text { otherwise. }\end{cases}
$$

Initially, no dissolved oxygen is present in the aquifer, and constant substrate and microbe concentrations of 6 and $.002 \mathrm{mg} / \mathrm{l}$, respectively, are assumed. Values for porosity, longitudinal and transverse dispersion, and the reaction coefficients are given in Table 1. These values remain constant throughout all of the simulations described below. Moreover, for all of the runs described below, except where noted, an 82 by 25 point-centered nonuniform mesh was employed, and the transport time step was .5 days, while the reaction time step was .00625 days. 
In (19), we set $\rho_{b}=2 \mathrm{gm} / \mathrm{cm}^{3}$, and $K_{o c}=259.5 \mathrm{~cm}^{3} / \mathrm{g}$. Corresponding to assumptions 1-3 on $f_{o c}$ discussed above, we will consider five different functional forms for $f_{o c}$ :

I. $f_{o c} \equiv 0$, thus $R_{S} \equiv 1$.

II. $f_{o c} \equiv .001$, giving $R_{S} \equiv 2.73$.

III. $f_{o c}$ varying stochastically, with mean $1.2 * 10^{-4}$, standard deviation of $1.6 * 10^{-4}$, minimum of 0 , and maximum of $1.34 * 10^{-3}$. This results in a retardation factor varying between 1 and 3.3 , with mean 1.2 . The correlation length in this case is roughly 10 feet.

IV. foc varying stochastically, with mean $9.6 * 10^{-4}$, standard deviation of $9.6 * 10^{-4}$, minimum of $3 * 10^{-4}$ and maximum of $7.9 * 10^{-3}$. This results in a retardation factor varying between 1.5 and 14.667 , with mean value 2.7. Again, the correlation length is 10 feet.

V. $f_{o c}$ varying linearly with depth, with $f_{o c}=.5 \%$ at the top of the aquifer, and $f_{o c}=0$ at the bottom. This leads to a retardation factor varying linearly between 1 and 9.7 , and decreasing with depth. The arithmetic mean retardation factor is 5.35 . 


\begin{tabular}{c|c}
\hline$\phi$ & .3 \\
$\alpha_{l}$ & $.6 \mathrm{ft}$ \\
$\alpha_{t}$ & $.001 \mathrm{ft}$ \\
$\bar{k}$ & $1.7 /$ day \\
$F$ & 3 \\
$Y$ & .5 \\
$K_{S}$ & $.13 \mathrm{mg} / 1$ \\
$K_{O}$ & $.1 \mathrm{mg} / 1$ \\
$B_{g}$ & $2 * 10^{-5} \mathrm{mg} / 1 /$ day \\
$b$ & $.01 /$ day \\
\hline
\end{tabular}

Table 1: Values of Physical Parameters.

We first consider a homogeneous medium, where $K \equiv 1.9 * 10^{-3} \mathrm{~cm} / \mathrm{sec}$, and demonstrate the effects on transport and biodegradation of varying retardation spatially. In Figures 1a through 1e, we have contoured substrate concentration at time 250 days, for $f_{o c}$ as given in I through V, respectively. Examining these results, we see that larger values of $R_{S}$ result in slower flow of substrate, and varying $R_{S}$ spatially causes substantial perturbation of the concentration profile. In each case, a "reaction zone" develops, which moves with the flow, where $O$ and $S$ are nonzero, and biodegradation occurs. This zone is relatively narrow in these simulations, since line drive flow is modeled. For $R_{S}$ values close to 1 , the reaction zone is extremely thin at any fixed time, since dissolved oxygen and substrate move at roughly the same speed. In fact, the predominant mixing effect in this situation is physical dispersion. As $R_{S}$ gets larger, the zone widens. Dissolved oxygen moves at a faster 

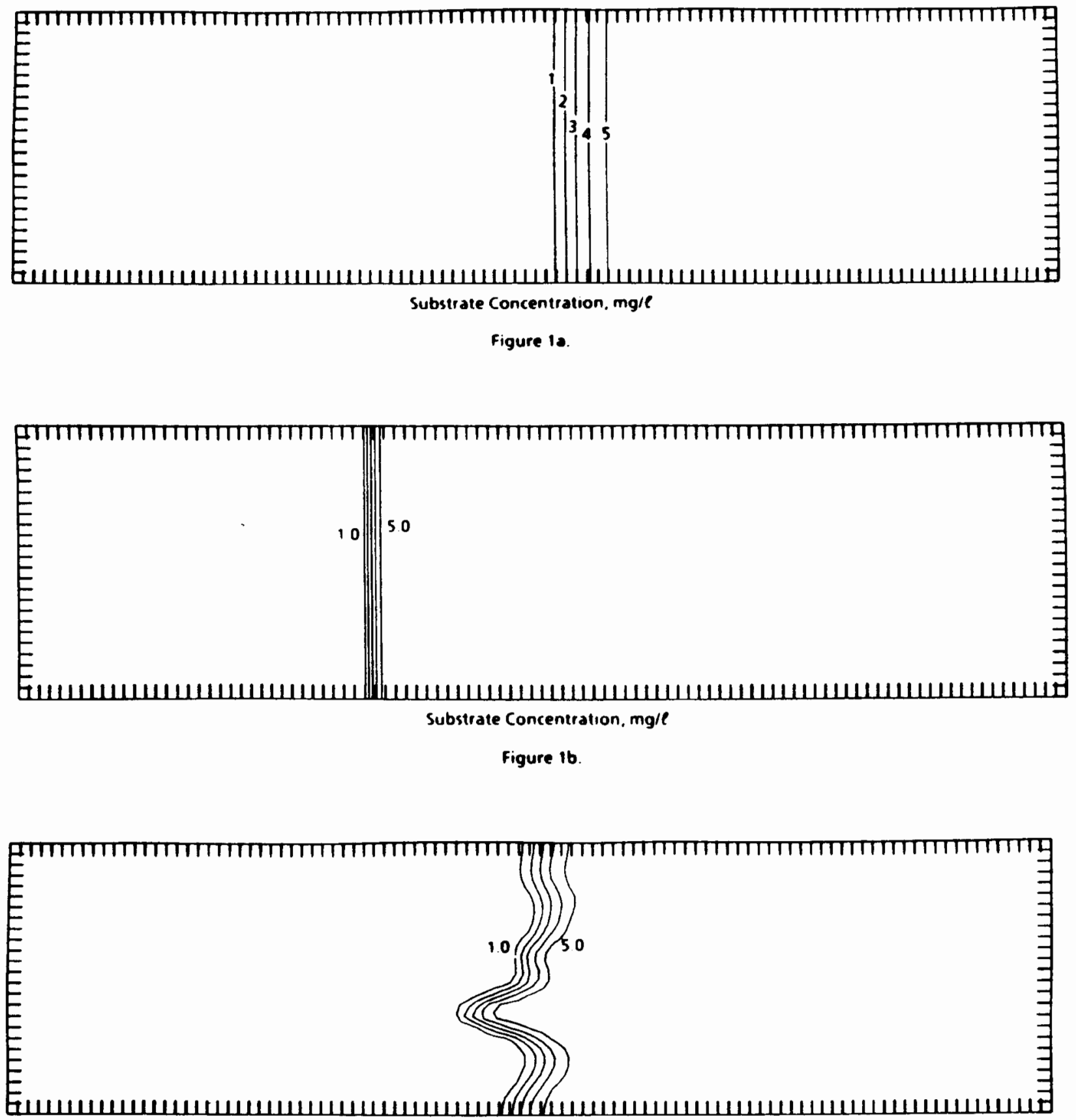

Substrate Concentration, mg/l

Figure ic 

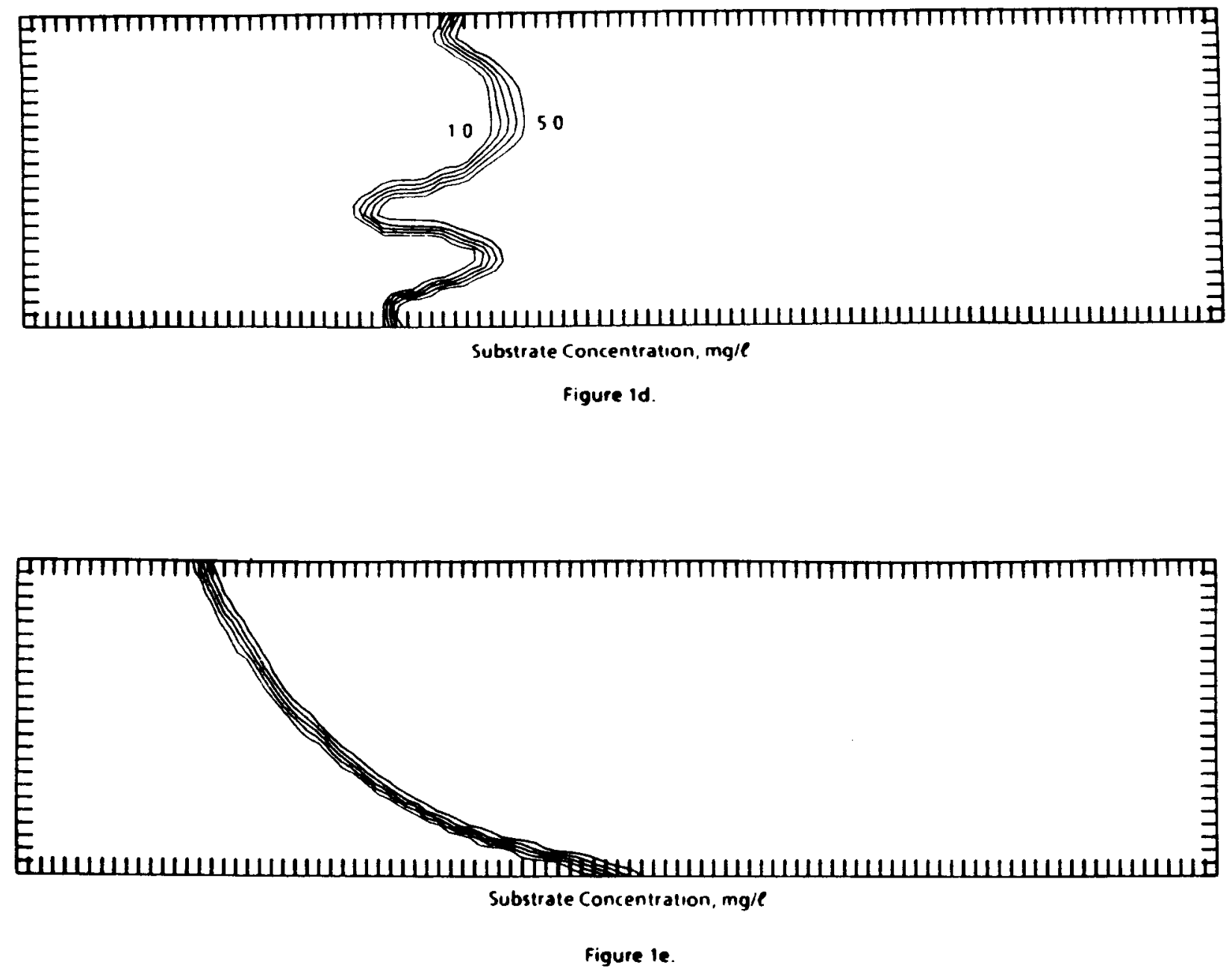
rate than substrate, and overtakes it, allowing for biodegradation to occur over a larger spatial region. However, the amount of biodegradation which can occur at any fixed point in space is actually reduced by increased adsorption. This dichotomy is reflected in (16) and will be examined in more detail below. We also note that, in the higher retardation cases II and IV, the substrate profile shows a steeper front than in the lower retardation cases I and III, compare Figures $1 b$ and $1 d$ with Figures $1 a$ and $1 c$. The reasons for this are two-fold. First, effective dispersion $\left(D / R_{S}\right)$ levels are lower in cases II and IV; moreover, more biodegradation is occurring at the leading edge of the front in these cases than in cases I and III, due to the increased overlapping of dissolved oxygen and substrate caused by retardation. Thus, the smooth edges of the front are removed, leaving a sharper profile. We will see this same effect in other situations below.

More information on these simulations is given in Figures 2 and 3. In Figure 2, we plot mass storage of substrate in solution at time $t$ (days) for all of the cases listed above; that is, we are plotting

$$
f(t)=\int_{\Omega} \phi S(x, t) d x
$$

Here, $f$ is in milligrams of substrate. Note that, in all cases, $f$ is a decreasing function of time, and $f$ increases with increasing retardation factor. The decrease in $f$ with time is due to biodegradation and flow of substrate out of $\Omega$; recall that we assume no substrate flows into the aquifer, since this boundary is rich in dissolved oxygen. The increase in $f$ with $R_{S}$ shows that, in all cases, the slower outflow of substrate caused by increasing adsorption rates dominates any increase in biodegradation due to overlapping of dissolved oxygen and retarded substrate. This is obvious from comparing the position of the fronts in Figures 1a-1e. A closer look at the relationship between adsorption and biodegradation is given in 
Figure 3. In this figure, we plot the amount of substrate mass biodegraded versus time, given by (see (16))

$$
g(t)=-\int_{0}^{t} \int_{\Omega} \phi R_{S}^{-1} R S(O, S, B) d x d t
$$

In this equation we have two competing terms, $R_{S}^{-1}$ and $R S$. For $R_{S}=1$, the overlap between dissolved oxygen and substrate is very narrow, causing $R S$ to be zero over a large portion of the space-time domain. For $R_{S}$ increasing but still close to one, the reaction zone grows, enlarging the area over which $R S$ is nonzero, and thus increasing $g$. Eventually though, $R_{S}^{-1}$ dominates, that is, a threshold retardation factor is reached where adsorption severely limits the amount of substrate mass biodegraded. This is verified in Figure 3 . For cases I-IV, $g$ increases with retardation (at any fixed time); however, in case $V$, which has the highest mean retardation factor, we see a decrease in mass biodegraded. In fact, $g$ in case $\mathrm{V}$ lies between the case III and case IV values. We found by numerical experimentation that, for this particular problem, $g$ increases with retardation up to $R_{S} \approx 3$, where adsorption begins to dominate, and from this point on, mass biodegraded decreases with increasing retardation. In general, this threshold retardation factor will vary with the soil conditions.

We next consider a more realistic scenario, with flow through a vertically stratified cross-section of a porous medium. A hydraulic conductivity field was generated which is representative of typical aquifers in the U. S. Gulf coastal region. In this field, the conductivity varies from $1 * 10^{-5}$ to $5 * 10^{-3} \mathrm{~cm} / \mathrm{sec}$, with arithmetic mean value of $1.9 *$ $10^{-3} \mathrm{~cm} / \mathrm{sec}$. A contour map of the hydraulic conductivity $K(x, y)$ is given in Figure 4 . In Figure 5, we show the substrate solution at 125 days with $R_{S} \equiv 1$ and no aerobic biodegradation, that is, we assume no dissolved oxygen is present in the system. In Figure 6 , 


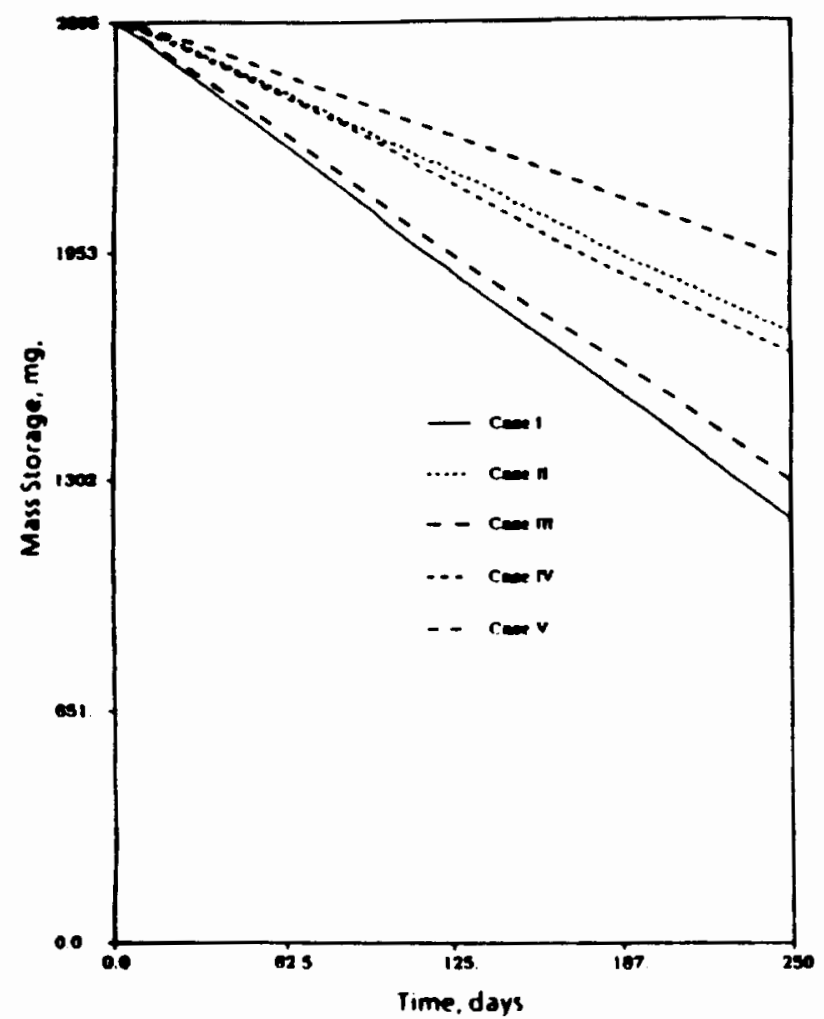

Figure 2: Mass storage (mg) vs. time (days) for cases $I-\mathrm{V}, K \equiv 1.9 * 10^{-3} \mathrm{~cm} / \mathrm{sec}$.

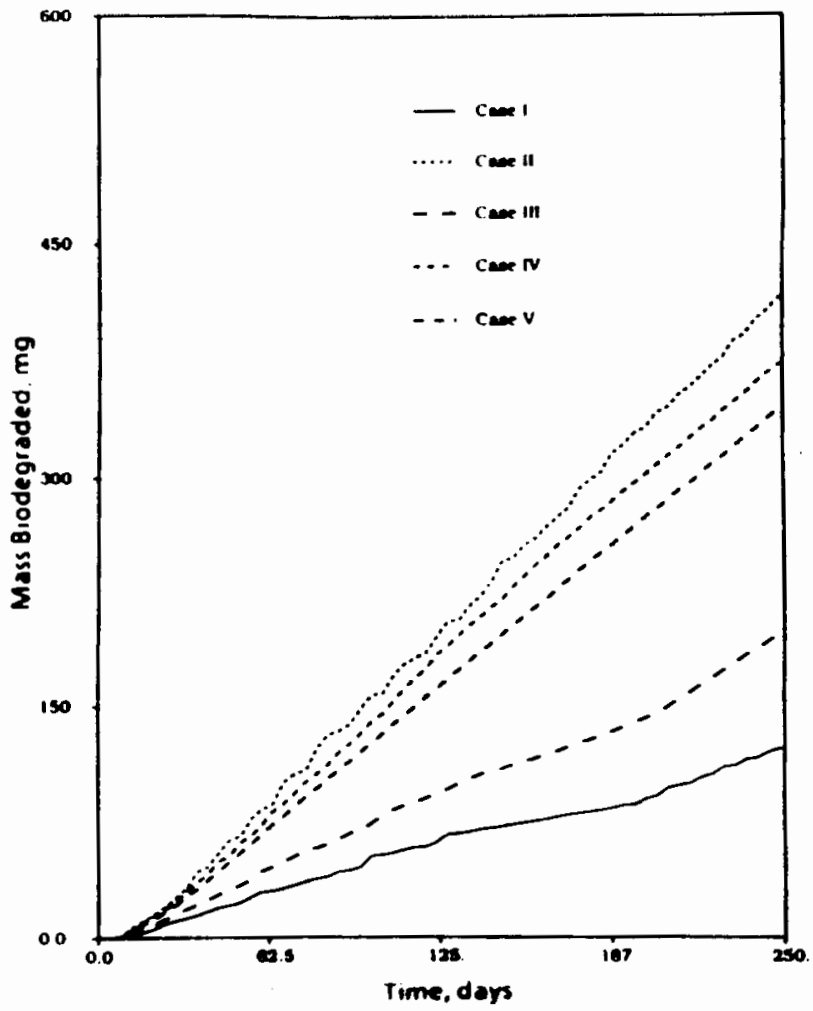

Figure 3: Mass biodegraded (mg) vs. time (days) for cases $\mathrm{I}-\mathrm{V}, K \equiv 1.9 * 10^{-3} \mathrm{~cm} / \mathrm{sec}$. 


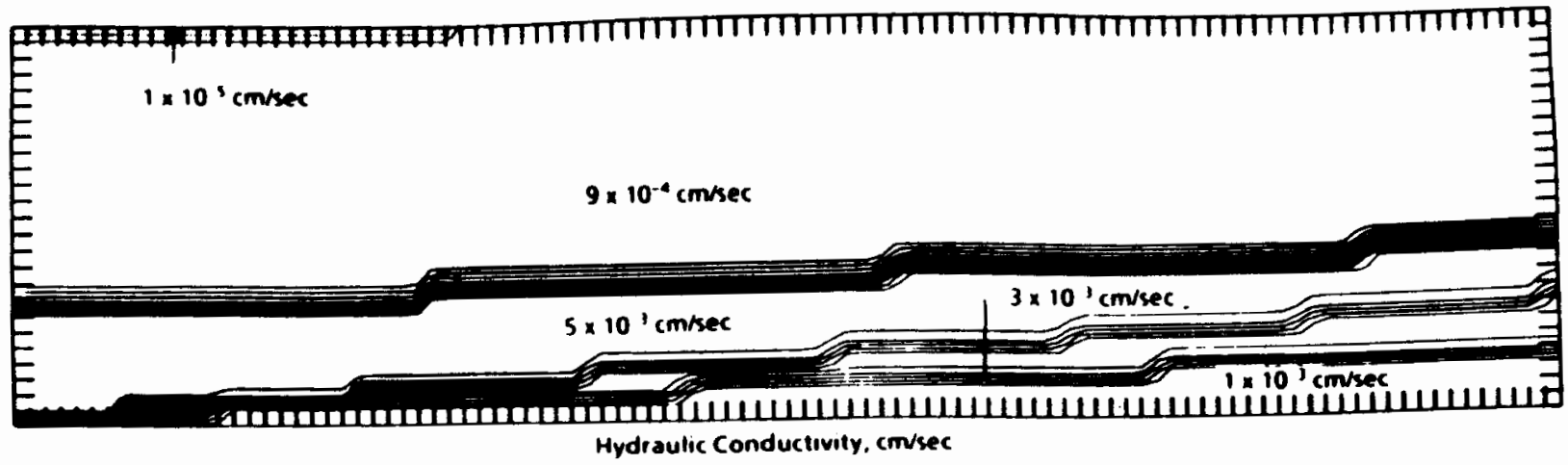

Ficure 4: Contour map of vertically stratified conductivity.

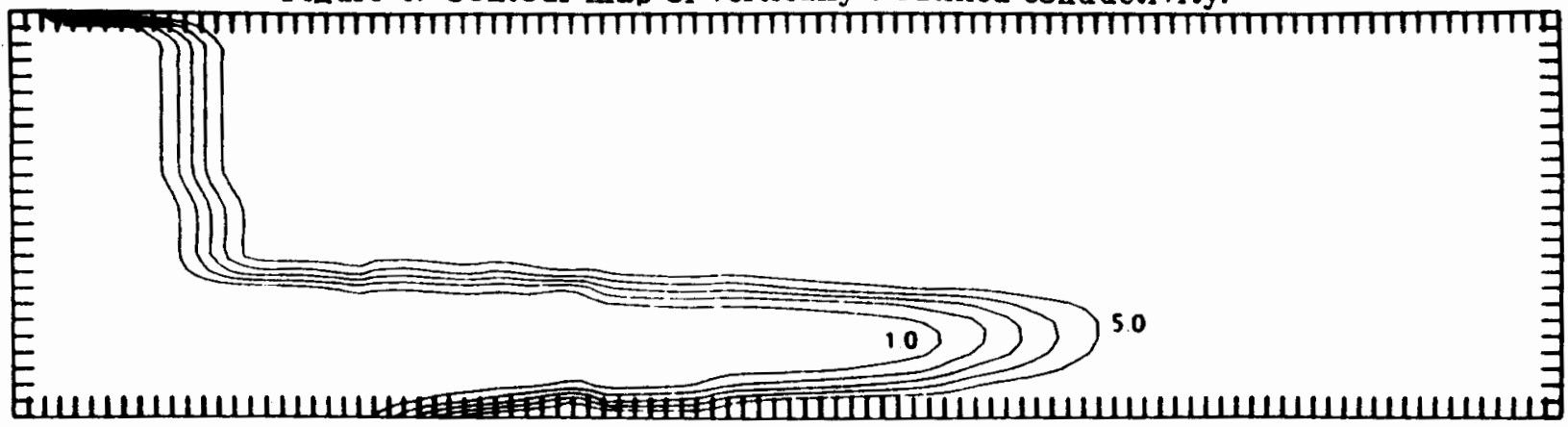

Figure 5: Substrate solution at 125 days, stratified medium, $R_{S} \equiv 1$, no biodegradation.

we show the same solution with biodegradation. Note that the protruding finger in these figures corresponds to flow in the higher hydraulic conductivity zone. Also, comparing Figures 5 and 6 , we note that little biodegradation has occurred, since physical dispersion is low and dissolved oxygen and substrate are moving at the same speed. In Figure 7 and Figure 8, we do the same comparison for $R_{S} \equiv 2.73$, that is, Case II above. We see that a substantial amount of biodegradation has occurred. These results agree with the results of

Figure 3 above. We also note that the biodegraded fronts are steeper than those without

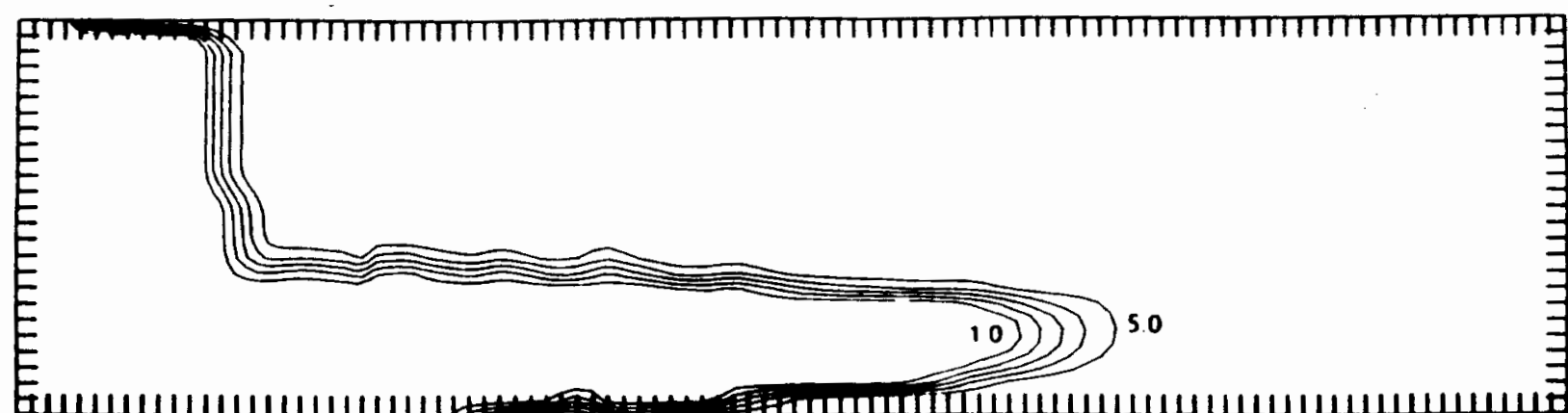

Figure 6: Substrate solution at 125 days, stratited medium, $R_{S} \equiv 1$, with biodegradation. 


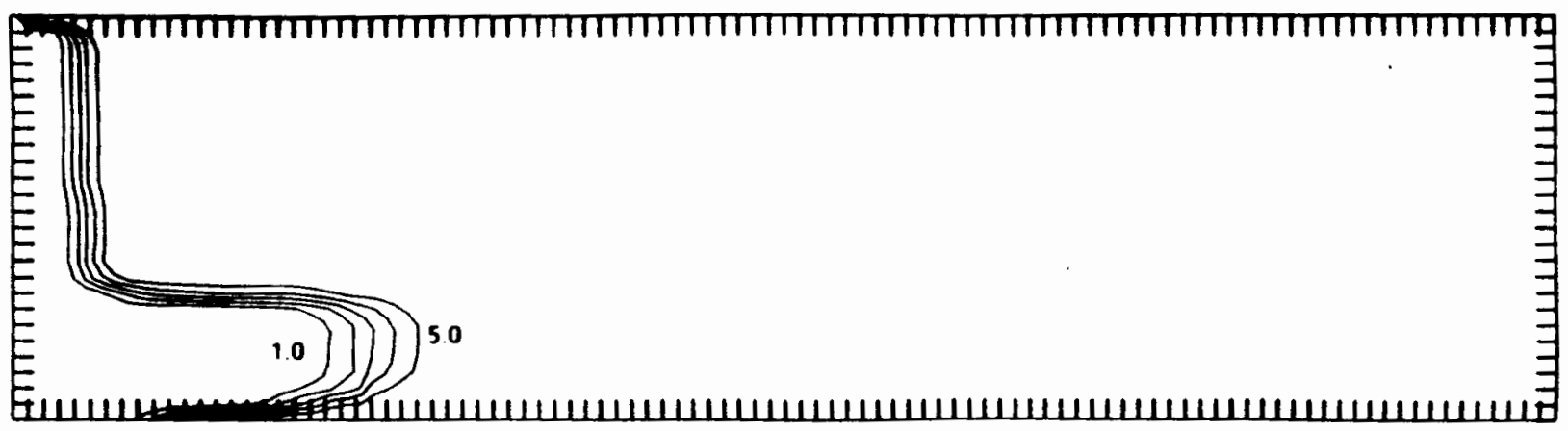

Substrate Concentration. mg/l

Figure 7: Substrate solution at 125 days, stratified medium, $R_{S} \equiv 2.73$, no biodegradation.

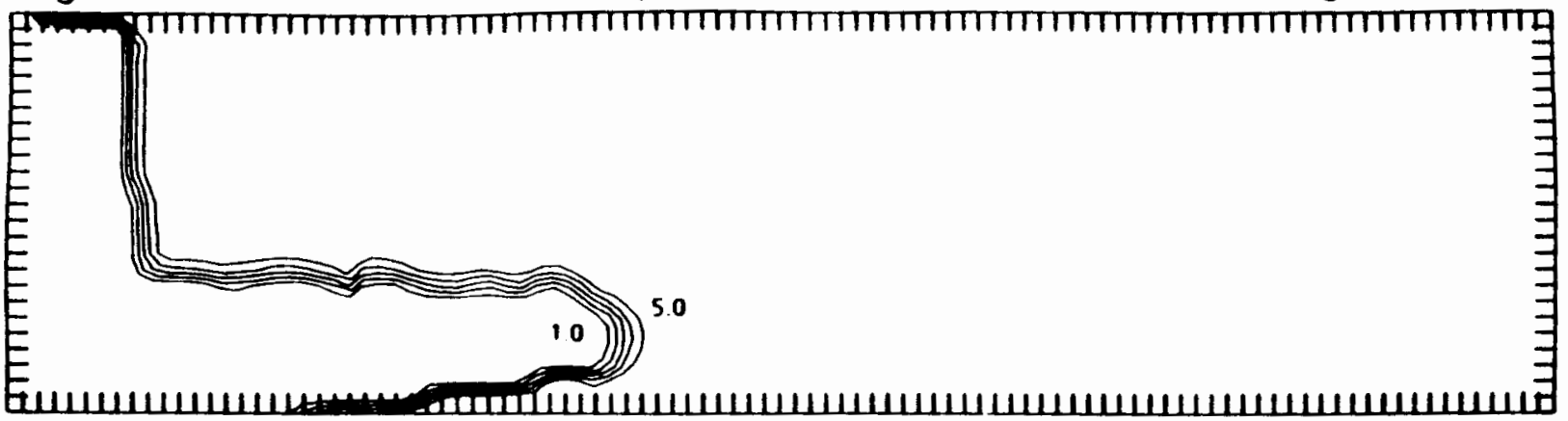

Figure 8: Substrate solution at 125 days, stratified medium, $R_{S} \equiv 2.73$, with biodegradation.

biodegradation.

Convergence of the substrate solution under mesh refinement is studied in Figures 9 and 10. Here we have taken the same physical data used to generate Figure 8 above and performed simulations with 82 by 48 and 91 by 70 nonuniform grids. As seen in these figures, the substrate front sharpens as the mesh is refined. However, the 82 by 48 and 91 by 70 solutions are virtually identical.

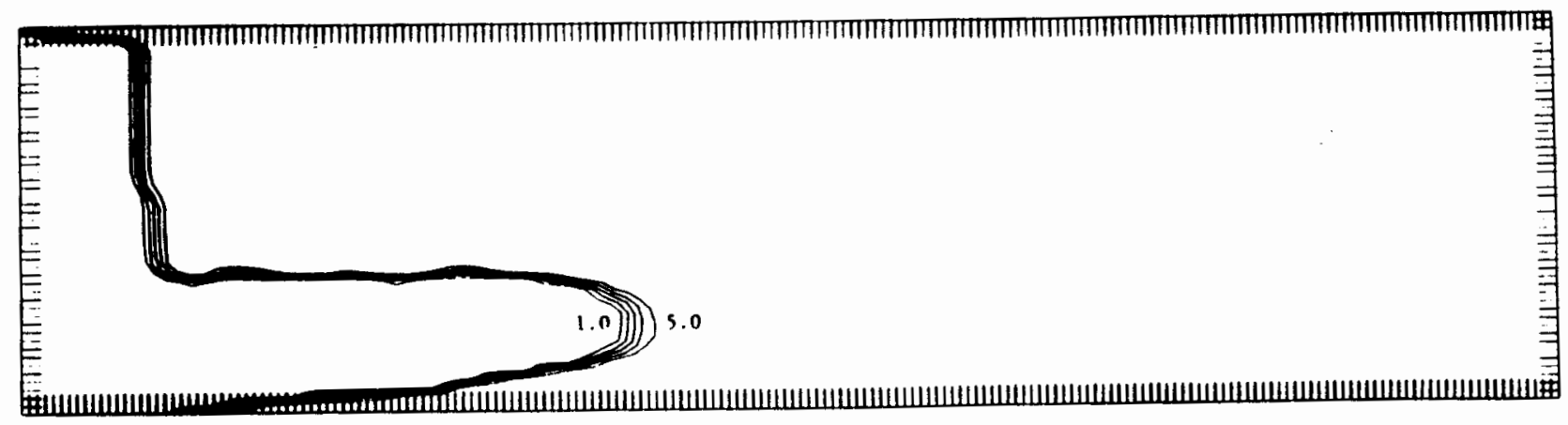

Figure 9: Substrate solution at 125 days, stratified medium, $R_{S} \equiv 2.73$, with biodegradation, 82 by 48 mesh. 


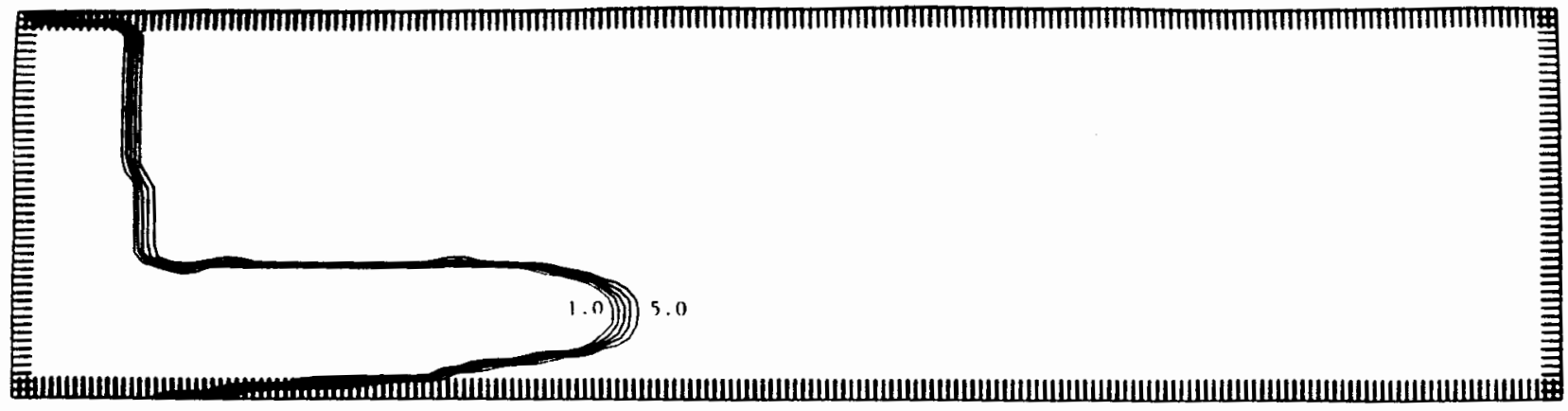

Figure 10: Substrate solution at 125 days, stratified medium, $R_{S} \equiv 2.73$, with biodegradation, 91 by 70 mesh.

The dissolved oxygen solution corresponding to the substrate solution in Figure 8 (stratified porous medium, $R_{S} \equiv 2.73$ ) is plotted in Figure 11. The figure shows that, as expected in this case, dissolved oxygen essentially fills the region void of substrate.

We have also considered the microbe solution for this case. Based on the mesh refinement study (Figures 8-10) above, the substrate solution for this problem is convergent on relatively coarse meshes. We have found by experimentation, however, that a truly accurate picture of the corresponding microbe solution requires very fine spatial mesh. In general, under the assumption of the Monod kinetics, the microbe solution is extremely sensitive to small variations in the hydrocarbon and oxygen solutions, at least for the values of reaction parameters chosen here. This sensitivity can be seen more clearly by the following argument. Holding substrate and dissolved oxygen concentrations fixed over a small time interval $[\tau, \tau+\epsilon]$, one can show, by solving (18) analytically, that,

$$
B(x, y, \tau+\epsilon)=e^{\alpha(x, y) \epsilon}\left[\frac{B_{g}}{\alpha(x, y)}+B(x, y, \tau)\right]-\frac{B_{g}}{\alpha(x, y)}
$$

where

$$
\alpha(x, y)=\bar{k} \cdot F \cdot\left(\frac{S(x, y)}{K_{S}+S(x, y)}\right) \cdot\left(\frac{O(x, y)}{K_{O}+O(x, y)}\right)-b
$$

Thus, for regions where $\alpha>0$, which is usually the case when both substrate and dissolved 
oxygen are present, the solution $B$ grows by an exponential factor. Thus, one can see that small variations in $\alpha$ can lead to large variations in the solution $B$.

In the stratified medium presented above, the predominant growth zones for microbes are the transition regions between higher and lower conductivity; these regions are also where $\alpha$ in (24) varies the most. The microbial growth is higher in these zones because the flow patterns dictate that substrate from the lower conductivity layers feeds into the higher conductivity layer, where dissolved oxygen is present. Eventually, the dissolved oxygen front in the lower conductivity zones catches up with substrate; however, in the transition regions $\alpha$ is positive for a substantial period of time, during which microbes grow exponentially. Since physical (especially transverse) dispersion in this case is assumed extremely low, the microbe solution exhibits very sharp "peaks" in these transition zones. This effect is exhibited in Figure 12. Here we plot the microbe solution at 125 days for the stratified porous medium with constant $R_{S}=2.73$, at $x=40$ feet, for three different meshes. The meshes used were 119 by 97,159 by 114 , and 164 by 155 , with most of the grid blocks concentrated in and around the higher conductivity layer. The solutions exhibit two very sharp peaks, corresponding to the two transition zones between higher and lower conductivity (see Figure 4). The peak becomes sharper with each mesh refinement, but the solutions appear to be converging. Inside the higher conductivity layer, more moderate microbial growth occurs, while outside these regions, microbes are essentially dormant. This phenomenon of microbial growth in transition zones between higher and lower hydraulic conductivity layers has also been observed in the laboratory [29].

In Figures 13, 14, and 15, we have plotted the substrate solutions at 125 days for the stratified porous medium, with $f_{o c}$ as given in III-V, respectively. Comparing these results 


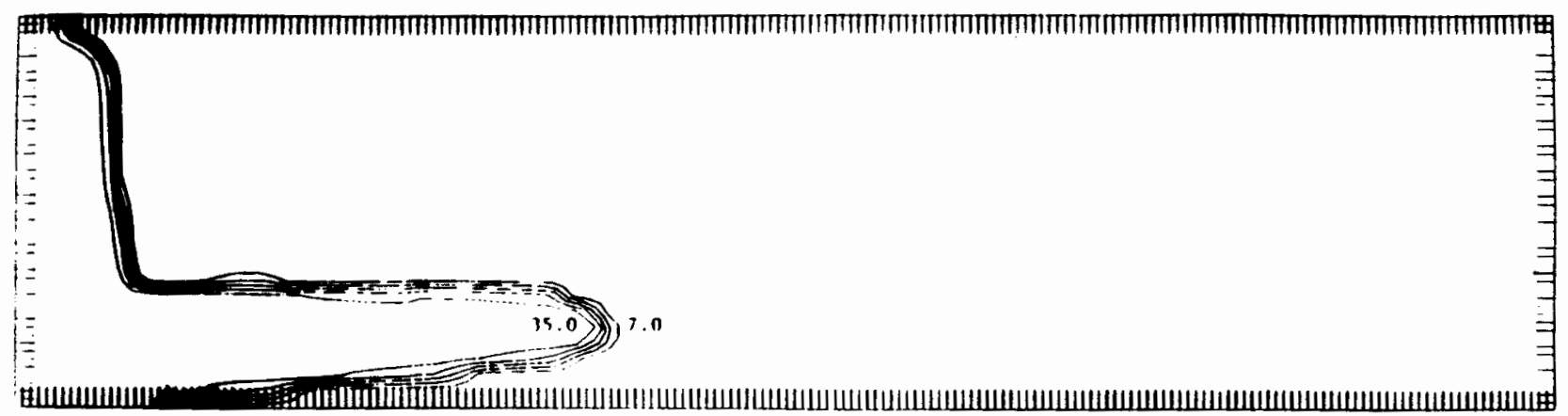

Figure 11: Dissolved oxygen solution at 125 days, corresponding to substrate solution in Figure 8, stratified medium, $R_{S} \equiv 2.73$.

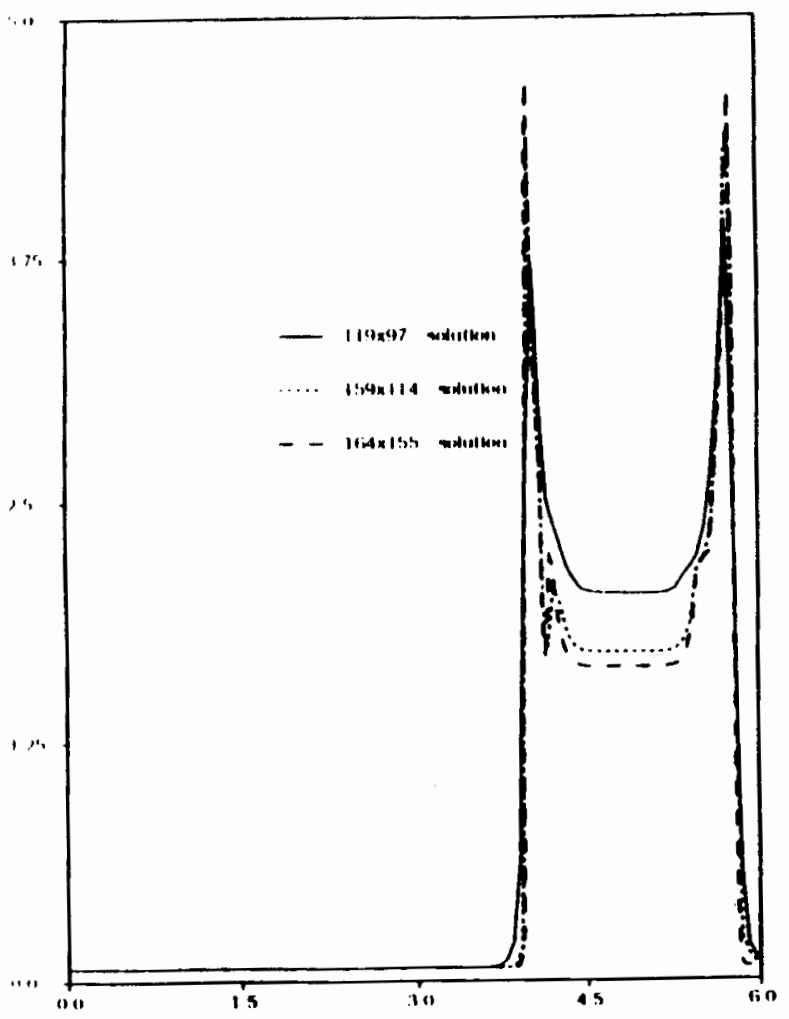

Figure 12: Microbe solution along $x=40$ feet at 125 days for three different meshes, stratified porous medium, $R_{S} \equiv 2.73$. 


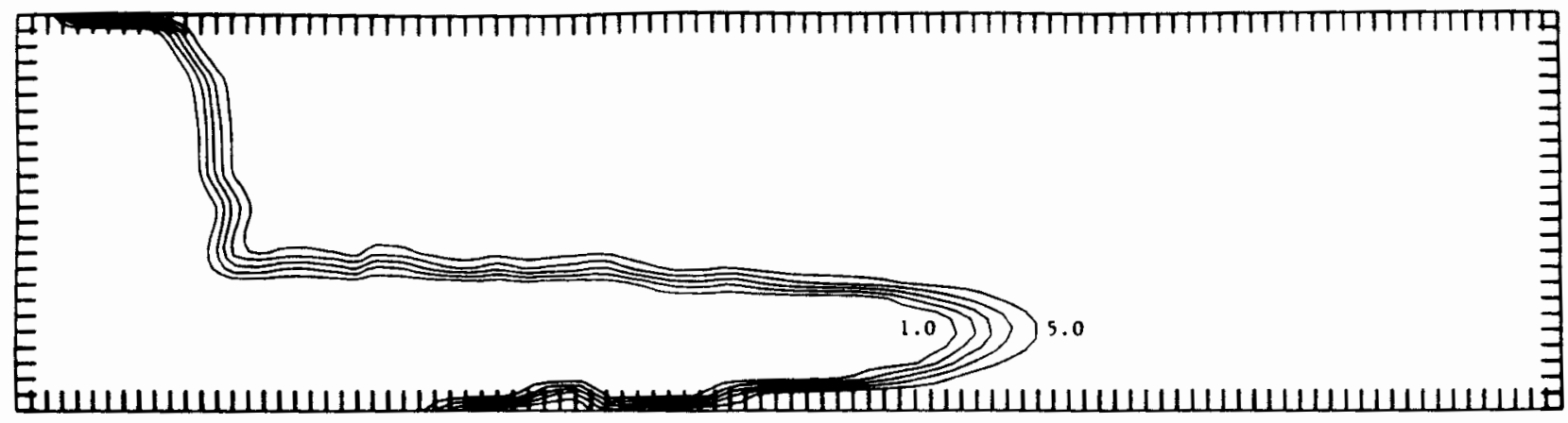

Figure 13: Substrate solution at 125 days, stratified medium, variable $f_{o c}$, mean $R_{S}=1.2$.

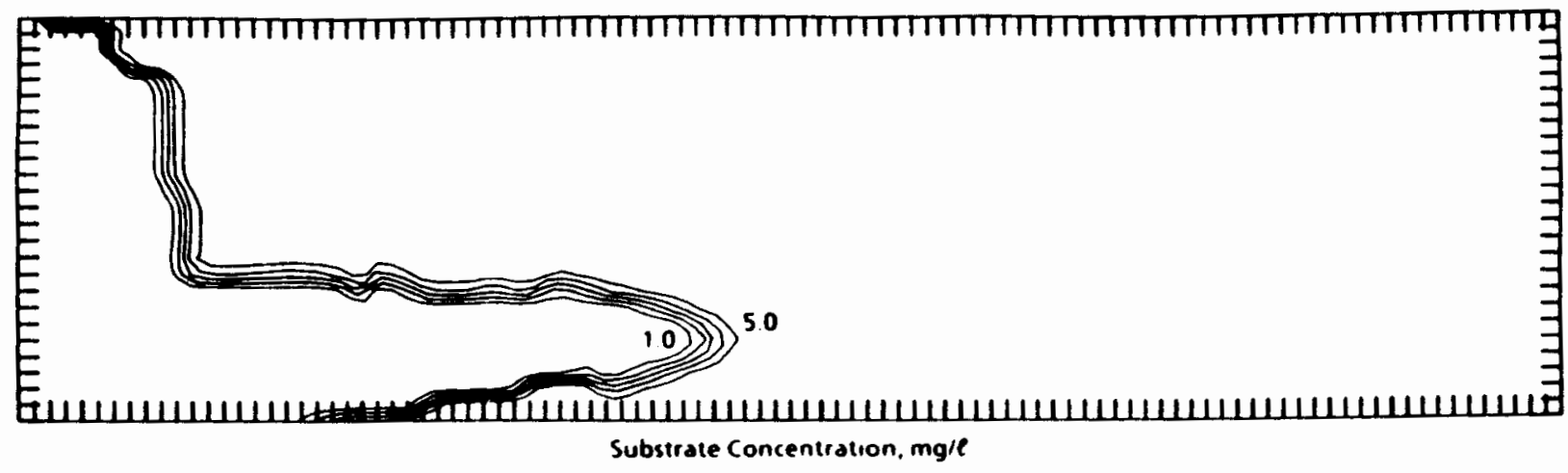

Figure 14: Substrate solution at 125 days, stratified medium, variable $f_{o c}$, mean $R_{S}=2.7$.

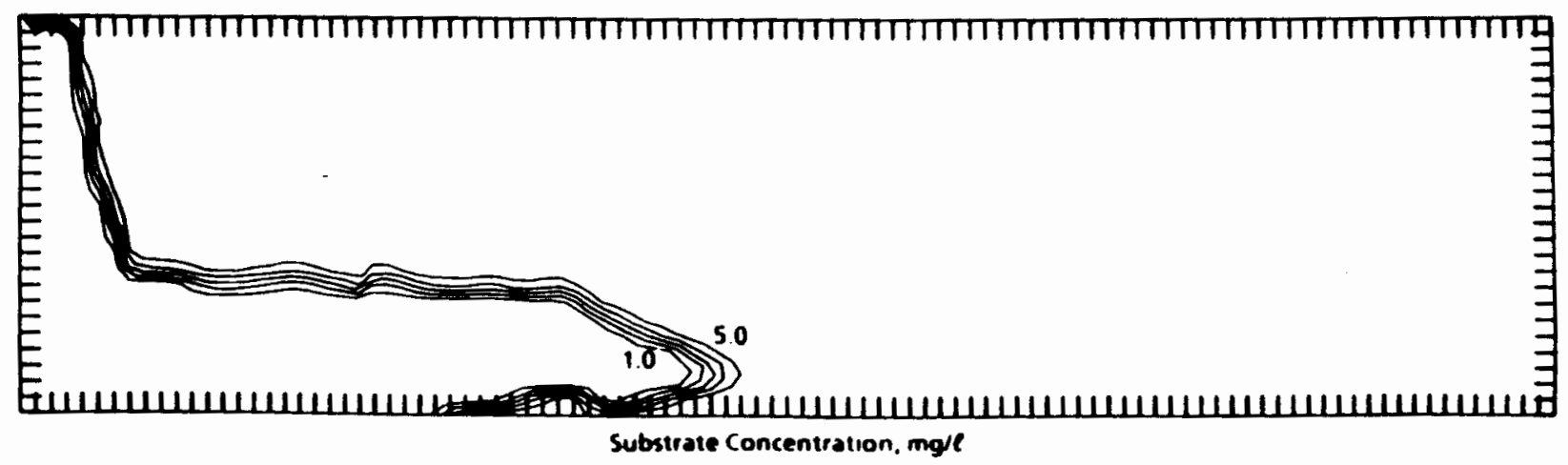

Figure 15: Substrate solution at 125 days, stratified medium, stratified $f_{o c}$. 


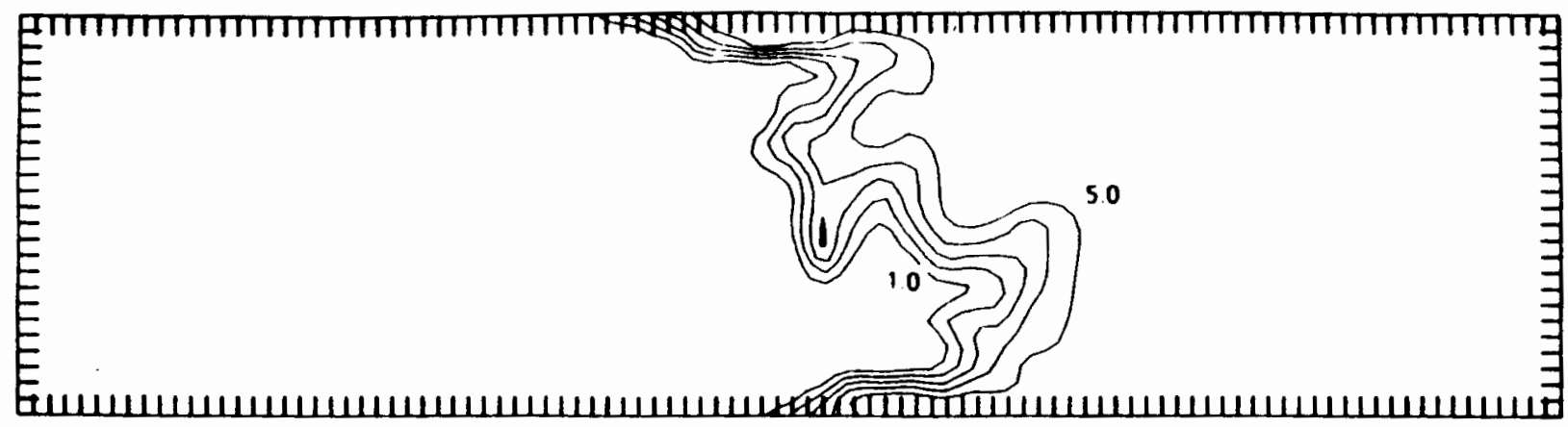

Figure 16: Substrate solution at 250 days, $K$ varying statistically, constant $f_{o c}, R_{S} \equiv 1$.

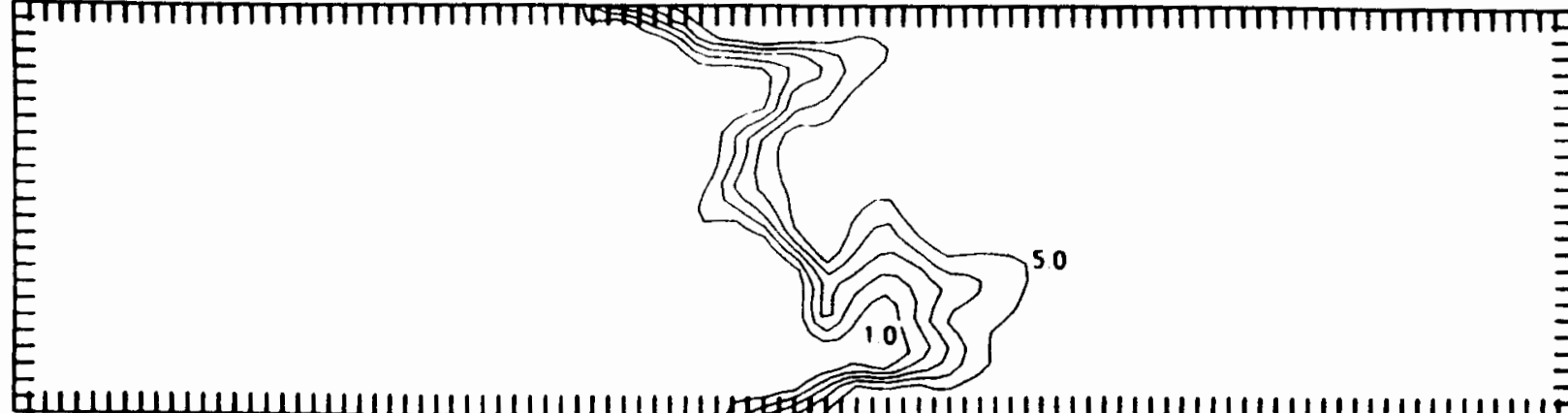

Figure 17: Substrate solution at 250 days, $K$ varying statistically, variable $f_{o c}$, mean $R_{S}=$ 1.2 .

to those given in Figures 6 and 8 , we see that, for these runs, the dominant effect on the flow is the high conductivity layer.

In our final set of simulations, we consider a conductivity field $K(x, y)$ generated statistically, again using the Moissis code. In these runs, the statistical mean of $K$ is $1.9 * 10^{-3}$ $\mathrm{cm} / \mathrm{sec}$, the minimum value is $3.9 * 10^{-4}$, the maximum value is $8.1 * 10^{-2}$, and the standard deviation is $3.07 * 10^{-3} \mathrm{~cm} / \mathrm{sec}$. The correlation length is 10 feet in the longitudinal direction and .24 feet in the transverse direction. We will again examine the relative effects of conductivity and organic carbon variations. First, the substrate solution for $f_{o c}=0$ at 250 days is plotted in Figure 16. We compare this solution to Figure 17, which corresponds to statistically varying $f_{o c}$ as described in III above, Next, the substrate solution for $f_{o c} \equiv .001$ at 250 days is plotted in Figure 18. We compare this to the substrate solution in Figure 19, which corresponds to IV above. Note that, while differences in the solutions are definitely 


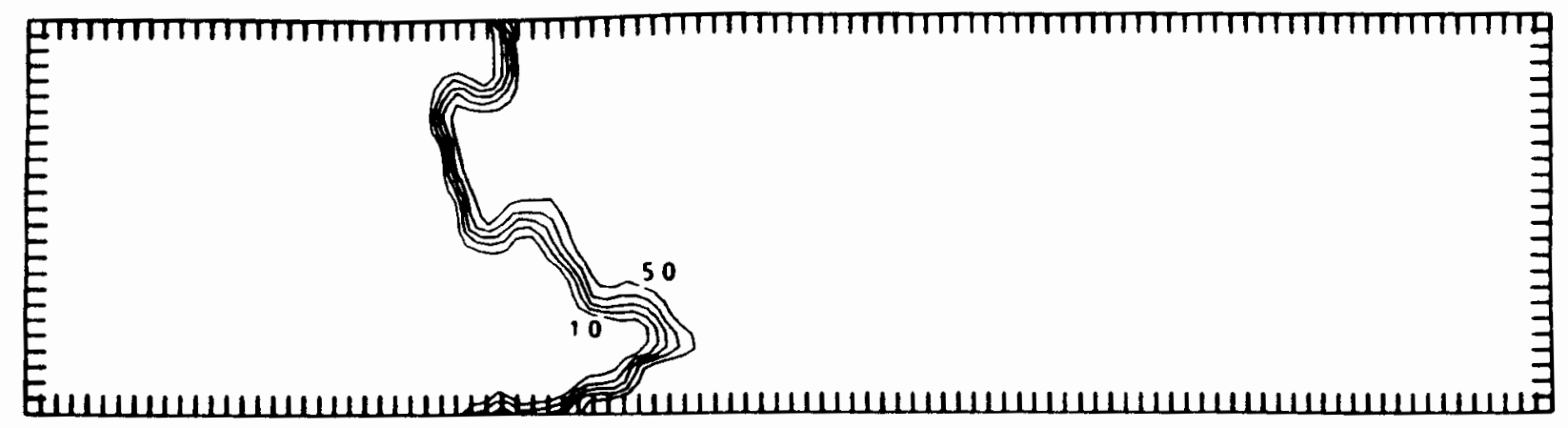

Figure 18: Substrate solution at 250 days, $K$ varying statistically, constant $f_{o c}, R_{S}=2.73$.

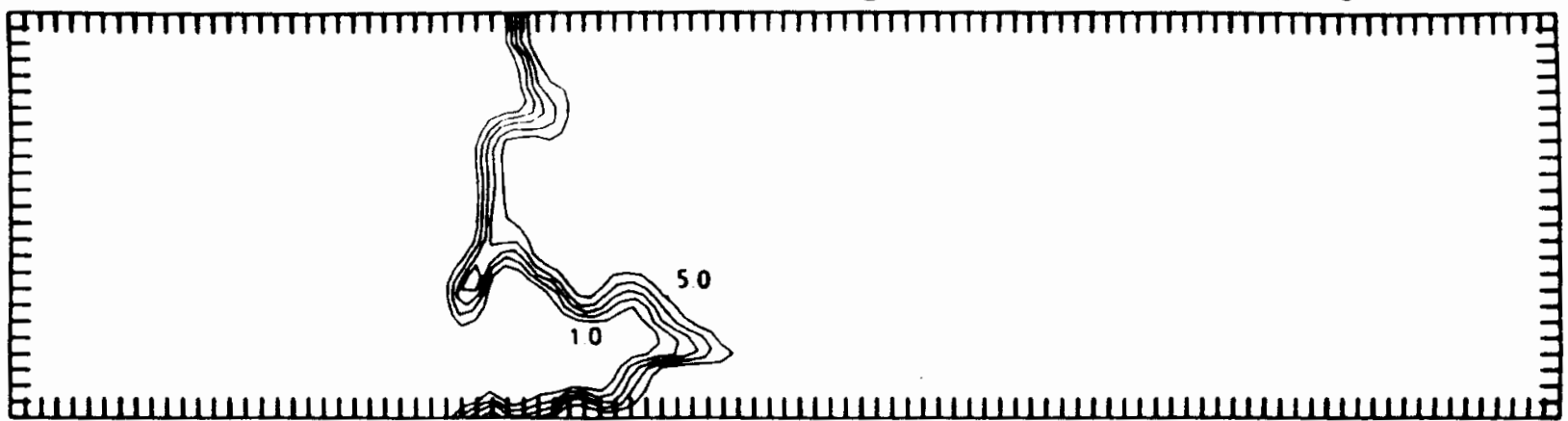

Figure 19: Substrate solution at 250 days, $K$ varying statistically, variable $f_{o c}$, mean $R_{S}=$ 2.7.

observed, qualitatively the solutions are very similar. Thus, conductivity variations still seem to be dominating factor. We now consider stratified $f_{o c}(V)$. In Figures $20-22$, we show the substrate solutions at 250,375 , and 500 days for this case. We see that these results are qualitatively similar to the result in Figure le above, where conductivity was constant. Thus, retardation variations seem to dominate in this situation.

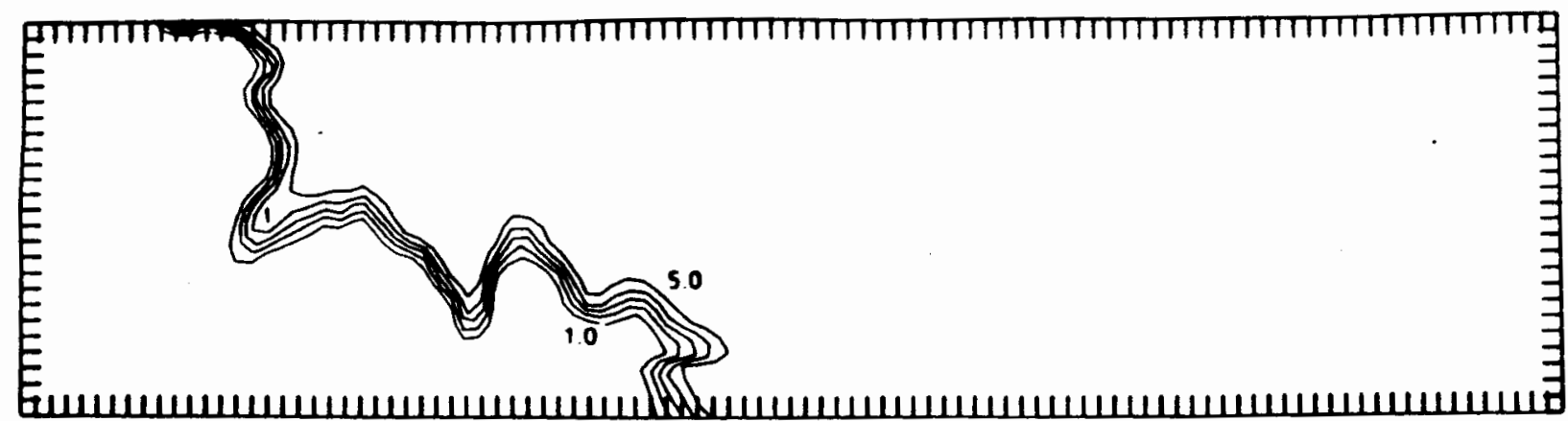

Figure 20: Substrate solution, $K$ varying statistically, stratified $f_{o c}, 250$ days. 


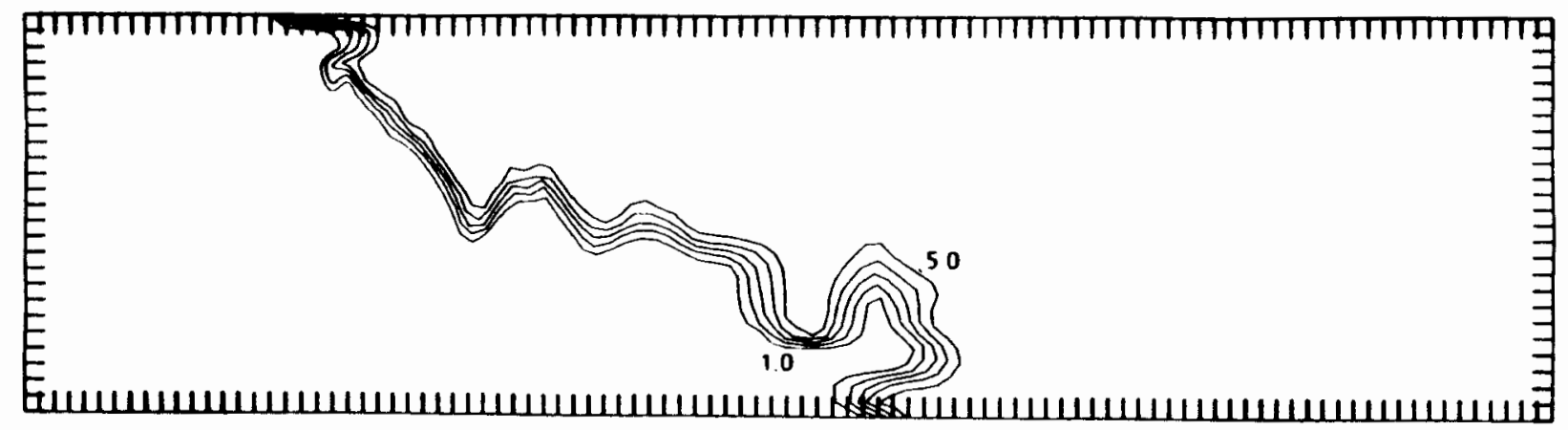

Figure 21: Substrate solution, $K$ varying statistically, stratified $f_{\text {oc }}, 375$ days.

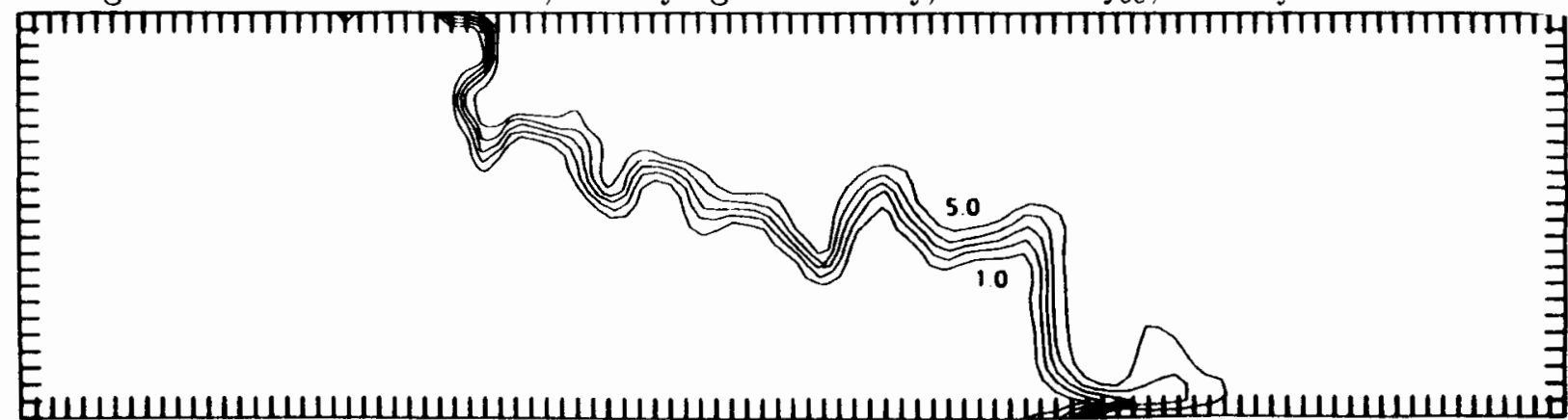

Figure 22: Substrate solution, $K$ varying statistically, stratified $f_{o c}, 500$ days.

\section{Conclusions}

An efficient numerical method has been presented which can be used to study the fate of soluble organics under the influence of enhanced biorestoration. This method treats systems of transport-biodegradation equations by operator splitting in time. Transport is approximated by a finite element modified method of characterisics. The biodegradation terms are split from the transport terms and treated as a system of ordinary differential equations. 28 The method has been applied to a typical enhanced biorestoration scenario, where dissolved oxygen is introduced into a contaminated aquifer. The effects of spatially varied hydraulic conductivity and organic carbon content (sorption) on the fate of soluble organics has been studied. We now summarize the results of this study, which are particular to the cases considered and to the mathematical model, but may also give insight to other practical problems of interest. 
We assumed an aquifer uniformly rich in organic carbon and substrate, and introduced dissolved oxygen to the system through an inflow boundary. Thus, a reaction zone developed, whose width depended on the levels of diffusion, dispersion, and substrate retardation. We assumed fixed diffusion and dispersion, and studied only the effects of retardation. When the retardation factor was high, dissolved oxygen moved at a faster rate than substrate, and overtook it, allowing for biodegradation to occur over a larger spatial region. In addition, sharper substrate concentration fronts were observed. The following two mechanisms caused these sharp fronts to develop. First, effective dispersion $\left(D / R_{S}\right)$ was decreased with increased retardation. Second, due to the increased overlapping of dissolved oxygen and substrate, the smooth edges of the substrate front were removed by biodegradation, leaving a sharper profile.

The simulations on the interactions between adsorption and biodegradation showed that, within a certain range, higher adsorption rates resulted in more substrate mass biodegraded. However, when the retardation factor increased above a threshold level, the substrate mass biodegraded decreased with increasing adsorption rates. This threshold level should vary with the soil conditions. When the sorption rate increased past this level, a significant amount of substrate mass was adsorbed and became unavailable to microorganisms.

Based on several numerical experiments, spatially varying hydraulic conductivity and organic carbon content had significant influence on solute transport. Not surprisingly, however, the dominant effect on the distribution of soluble organics in most cases was the spatial pattern of the hydraulic conductivity field.

Finally, in a vertically stratified porous medium with a higher conductivity layer sandwiched between regions of lower conductivity, the growth of microorganisms was predomi- 
nant in the transition areas between higher and lower hydraulic conductivity. This occurred because dissolved oxygen quickly filled the higher conductivity layer, and substrate was continually fed into this layer from the regions of lower conductivity.

Acknowledgments: The second author acknowledges the support of the National Science Foundation, Grant No. DMS-8807257, the National Center for Supercomputing Applications (NCSA) at the University of Ilinois in Urbana-Champaign, and the National Science Foundation Center for Research in Parallel Computation. Some of the computer simulations described above were run on the CRAY X-MP/48 at NCSA. The third author acknowledges the support of the Department of Energy, Grant No. DE-FG05-88ER-25060. The authors would also like to acknowledge the contributions of David Moissis and Mandri Obeyesekere to the preparation of this work.

\section{References}

[1] Borden, R. C. and P. B. Bedient, Transport of dissolved hydrocarbons influenced by oxygen-limited biodegradation 1. Theoretical development, Water Resour. Res. 22, pp. 1973-1982, 1986.

[2] Celia, M. A., J. S. Kindred, and I. Herrera, Contaminant transport and biodegradation, 1. A numerical model for reactive transport in porous media, Water Resour. Res. 25, pp. 1141-1148, 1989.

[3] Chiang, C. Y., J. P. Salanitro, E. Y. Chai, J. D. Colthart, and C. L. Klein, Aerobic biadegradation of benzene, toluene, and xylene in a sandy aquifer-data analysis and 
computer modelling, Ground Water 27, pp. 823-834, 1989.

[4] Chiang, C. Y., M. F. Wheeler, and P. B. Bedient, A modified method of characteristics technique and a mixed finite element method for simulation of groundwater solute transport, Water Resour. Res. 25, pp. 1541-1549, 1989.

[5] Dawson, C. N., T. F. Russell, and M. F. Wheeler, Some improved error estimates for the modified method of characteristics, SIAM J. Numer. Anal. 26, pp. 1487-1512 (1989).

[6] Dawson, C. N., M. F. Wheeler and P. B. Bedient, Numerical modeling of subsurface contaminant transport with biodegradation kinetics, Proceedings of National Water Wells Meeting, Houston, Texas, 1987, pp. 329-344.

[7] Dawson, C. N., M. F. Wheeler, T. M. Nguyen, and S. W. Poole, Simulation of hydrocarbon biodegradation in groundwater, CRAY Channels 8, 3, pp. 14-19, 1986.

[8] Dawson, C. N., M. F. Wheeler, T. M. Nguyen, and S. W. Poole, Simulation of subsurface contaminant transport with biodegradation kinetics, Proceedings of Third International Symposium on Science and Engineering on CRAY Supercomputers, Mendota Heights, Minnesota, pp. 75-86, 1987.

[9] Douglas, J. Jr., R. E. Ewing, and M. F. Wheeler, Approximation of the pressure by a mixed finite element method in the simulation of miscible displacement, RAIRO Anal. 17, pp. 17-33, 1983.

[10] Douglas, J. Jr. and T. F. Russell, Numerical methods for convection-dominated diffusion problems based on combining the method of characteristics with finite element or finite difference procedures, SIAM J. Numer. Anal. 19, pp. 871-885, 1982. 
[11] Douglas, J., T. F. Dupont, H. H. Rachford, and M. F. Wheeler, Local $H^{-1}$ Galerkin and adjoint local $H^{-1}$ Galerkin procedures for elliptic equations, RAIRO Anal. Numer. 11, pp. 3-12, 1977.

[12] Freeze, R. A. and J. A. Cherry, Groundwater, Prentice-Hall, Englewood Cliffs, New Jersey, 1979.

[13] Karickhoff, S. W., D. S. Brown, and T. A. Scott, Sorption of hydrophobic pollutants on natural sediments, Water Research 13, pp. 241-248, 1979.

[14] Kindred, J. S. and M. A. Celia, Contaminant transport and biodegradation 2. Conceptual model and test simulations, Water Resour. Res. 25, pp. 1149-1159, 1989.

[15] Konikow, L. F. and J. D. Bredeheoft, Computer model of two-dimensional solute transport and dispersion in groundwater. Automated data processing and computations. Techniques of Water Resources Investigations of the United States Geological Survey, Washington, D. C., 1978.

[16] MacQuarrie, K. T. B., E. A. Sudicky, and E. O. Frind, Simulation of biodegradable organic contaminants in groundwater 1, numerical formulation in principal directions, Water Resour. Res. 26, pp. 207-222.

[17] MacQuarrie, K. T. B. and E. A. Sudicky, Simulation of biodegradable organic contaminants in groundwater 2, plume behavior in uniform and random flow fields, Water Resour. Res. 26, pp. 223-240. 
[18] Miller, M. M., S. P. Wasik, G. L. Huang, W. Y. Shiu, and D. Mackay, Relationship between octanol-water partition coefficient and aqueous solubility, Environmental Science and Technology 19, pp. 522-529, 1985.

[19] Moissis, David, Simulation of viscous fingering during miscible displacement in nonuniform porous media, Ph. D. Thesis, Rice University, 1988.

[20] Molz, F. J., M. A. Widdowson, and L. D. Benefield, Simulation of microbial growth dynamics coupled to nutrient and oxygen transport in porous media, Water Resour. Res. 22, pp. 1207-1216, 1986.

[21] Nakata, M., A. Weiser, and M. F. Wheeler, Some superconvergence results for mixed finite element methods for elliptic problems on rectangular domains, MAFELAP Proceedings V, J. A. Whiteman, ed., Academic Press, New York, pp. 367$390,1985$.

[22] Raymond, R. L., Jamison, V. W., and Hudson, J. D., Final report on beneficial stimulation of bacterial activity in ground water containing petroleum products, Committee on Environmental Affairs, Americal Petroleum Institute, Washington, D. C., 1975.

[23] Rifai, H.S., Numerical techniques for modeling in situ biorestoration and biodegradation of organic contaminants in ground water, Ph. D. Thesis, Rice University, 1989.

[24] Rittman, B. E. and P. L. McCarty, Model of steady state biofilm kinetics, Biotech. Bioeng. 22, pp. 2343-2357, 1980. 
[25] Russell, T. F., Time-stepping along characteristics with incomplete iteration for a Galerkin approximation of miscible displacement in porous media, SIAM J. Numer. Anal. 22, pp. 970-1013, 1985.

[26] Russell, T. F., M. F. Wheeler, and C. Y. Chiang, Large-scale simulation of miscible displacement, Proceedings of SEG/SIAM/SPE Conference on Mathematical and Computational Methods in Seismic Exploration and Reservoir Modeling, W. E. Fitzgibbon, ed., Society for Industrial and Applied Mathematics, Philadelphia, pp. 85-107, 1986.

[27] Thomas, J. M., M. D. Lee, P. B. Bedient, R. C. Borden, L. W. Canter, and C. H. Ward, Leaking underground storate tanks: remediation with emphasis on in situ biorestoration, Environmental Protection Agency, 600/2-87,008, January, 1987.

[28] United States Department of Energy, Site-directed subsurface environmental initiative, five year summary and plan for fundamental research in subsoils and in groundwater, FY1989-FY1993, DOE/ER 034411, Office of Energy Research, April 1988.

[29] United States Department of Energy, Review of intermediate-scale experiments for subsurface microbiology and chemistry, DOE/ER-0383, Proceedings of an OHER/DOE Sponsored Workshop at University of Delaware's Virden Center, April 12-14, 1987.

[30] M. F. Wheeler and C. N. Dawson, An operator-splitting method for advection-diffusionreaction problems, MAFELAP Proceedings VI, J. A. Whiteman, ed., Academic Press, pp. $463-482,1988$.

[31] M. F. Wheeler, C. N. Dawson, P. B. Bedient, C. Y. Chiang, R. C. Borden, and H. S. Rifai, Numerical simulation of microbial biodegradation of hydrocarbons in groundwa- 
ter, Proceedings AGWSE/IGWMCH Conference on Solving Ground Water Problems with Models, National Water Wells Association, pp. 92-108, 1987.

[32] Widdowson, M. A., F. J. Molz, and L. D. Benefield, Development and application of a model for simulating microbial growth dynamics coupled to nutrient and oxygen transport in porous media, Proceedings AGWSE/IGWMCH Conference on Solving Ground Water Problems with Models, National Water Wells Association, pp. 28-51, 1987. 
